\title{
Growth mode and strain evolution during InN growth on GaN(0001) by molecular-beam epitaxy
}

\author{
Y. F. Ng, Y. G. Cao, M. H. Xie, ${ }^{\text {a) }}$ X. L. Wang, ${ }^{\text {b) }}$ and S. Y. Tong ${ }^{\text {c) }}$ \\ Department of Physics and HKU-CAS Joint Laboratory on New Materials, The University of Hong Kong, \\ Pokfulam Road, Hong Kong
}

(Received 14 August 2002; accepted 25 September 2002)

\begin{abstract}
Epitaxial growth of $\mathrm{InN}$ on $\mathrm{GaN}(0001)$ by plasma-assisted molecular-beam epitaxy is investigated over a range of growth parameters including source flux and substrate temperature. Combining reflection high-energy electron diffraction (RHEED) and scanning tunneling microscopy (STM), we establish a relationship between film growth mode and the deposition condition. Both two-dimensional (2D) and three-dimensional (3D) growth modes of the film are observed. For 2D growth, sustained RHEED intensity oscillations are recorded while STM reveals 2D nucleation islands. For 3D growth, less than three oscillation periods are observed indicating the StranskiKrastanov (SK) growth mode of the film. Simultaneous measurements of (reciprocal) lattice constant by RHEED suggest a gradual relaxation of the strain in film, which commences during the first bilayer (BL) deposition and almost completes after 2-4 BLs. For SK growth, 3D islanding initiates after the strain has mostly been relieved, presumably by dislocations, so the islands are likely strain free. (C) 2002 American Institute of Physics. [DOI: 10.1063/1.1523638]
\end{abstract}

In optoelectronic and microelectronic applications of group-III nitrides, heterostructures incorporating $\mathrm{InGaN}$ and GaN epilayers are important ingredient in, e.g., lasers and high-speed transistors. ${ }^{1,2}$ Due to some inherent problems associated with lattice mismatch and film stability, etc., heteroepitaxial growth of high quality $\mathrm{InGaN}$ on $\mathrm{GaN}$ has been shown difficult. ${ }^{3,4}$ For instance, new defects form in epitaxial InGaN films while In composition also fluctuates. ${ }^{5}$ Threedimensional (3D) growth mode induced by strain leads to rough surfaces, while the 3D islands may also be useful for realizing quantum dots. ${ }^{2}$ Depending on application, both two-dimensional (2D) and three-dimensional morphologies are desirable, so it is important to establish conditions under which the desired morphology is achieved and to learn the mechanisms by which strain in film is accommodated or relieved.

Despite recent research on InGaN alloys, less is known regarding epitaxial growth of binary $\mathrm{InN}$ on $\mathrm{GaN}^{6-8}$ In this letter, we report on the growth behavior of $\mathrm{InN}$ on $\mathrm{GaN}$ during molecular beam epitaxy (MBE). Specifically, we reveal a dependence of growth mode on the deposition condition (temperature and flux) and show strain evolution as growth proceeds.

The MBE system is equipped with a radio-frequency (13.56 MHz) plasma unit (Oxford Applied Research, CARS$25)$, generating reactive nitrogen $(\mathrm{N})$ species (atoms or ions) from $\mathrm{N}_{2}$ gas. Knudsen effusion cells are used for elemental sources such as gallium (Ga) and indium (In). The effective flux of the reactive nitrogen species is in the range of $0.68-2.25 \times 10^{14} \mathrm{~cm}^{-2} \mathrm{~s}^{-1}$, giving rise to a film growth rate

\footnotetext{
a) Author to whom correspondence should be addressed; electronic mail: mhxie@hkusua.hku.hk

${ }^{b)}$ Permanent address: Institute of Semiconductors, Chinese Academy of Sciences, Beijing 100083, People's Republic of China.

${ }^{c}$ Present address: Department of Physics and Materials Science, City University of Hong Kong, Kowloon, Hong Kong.
}

of 0.03-0.1 bilayers (BLs) $\mathrm{s}^{-1}$ (i.e., $0.078-0.26 \AA^{-1}$ for $\mathrm{GaN})$. Our experiments suggest that growth mode is not significantly affected by the absolute values of the flux, whereas the ratio between $\mathrm{In}$ and $\mathrm{N}$ fluxes has a dramatic effect on morphology and film growth mode. Prior to InN deposition, a $\mathrm{GaN}$ buffer layer of about $1 \mu \mathrm{m}$ thick is grown on $\mathrm{SiC}(0001)-(\sqrt{3} \times \sqrt{3}) R 30^{\circ}$ at temperatures $600-650^{\circ} \mathrm{C}$ and under the excess-Ga flux condition $(\mathrm{Ga} / \mathrm{N} \sim 2)$. This procedure results in an atomically flat $\mathrm{GaN}$ surface showing the Ga polarity. ${ }^{9}$ Furthermore, the surface is known to be covered by two layers of Ga atoms. ${ }^{10}$ To desorb such excess Ga layers, the sample is briefly annealed in $\mathrm{N}$ flux (closing the Ga source shutter), during which the RHEED patterns change from " $1 \times 1$," typical for a Ga-covered $\mathrm{GaN}(0001)$ surface, to " $2 \times 2$ " indicating a Ga-deficient surface. ${ }^{10} \mathrm{Im}$ mediately afterwards, the substrate temperature is lowered to $350-450{ }^{\circ} \mathrm{C}$ and $\mathrm{InN}$ deposition is followed. RHEED specular beam intensity is recorded, at the same time RHEED patterns in the [1 $\overline{1} 00]$ azimuth and the spacing between (01) and $(0 \overline{1})$ diffraction streaks are monitored. The RHEED operates at $10 \mathrm{kV}$ with an incident angle of $0.4^{\circ}$, corresponding to the out-of-phase diffraction condition. Thermally quenched surfaces of InN are examined by an ultrahigh vacuum scanning tunneling microscope (UHV-STM) operating under the constant current mode and at room temperature. For all STM measurements, the sample bias is $-2.0 \mathrm{~V}$ and the tunneling current is set at $0.1 \mathrm{nA}$.

Figure 1 shows the RHEED intensity oscillations during InN growth on $\mathrm{GaN}$ at (a) $450{ }^{\circ} \mathrm{C}$ and (b) $370{ }^{\circ} \mathrm{C}$, respectively. For (a), In/N flux ratio is 1.5 whereas for (b), it is 0.6. RHEED intensity oscillation during homoepitaxial growth of $\mathrm{GaN}$ is also given in Fig. 1 (trace c) for comparison. ${ }^{9}$ From the figure, two different growth behaviors of $\mathrm{InN}$ can be distinguished: 2D layer-by-layer growth for (a) and Stranski-Krastanov (SK) mode for (b). The former is evidenced by the sustained RHEED oscillations while the latter 


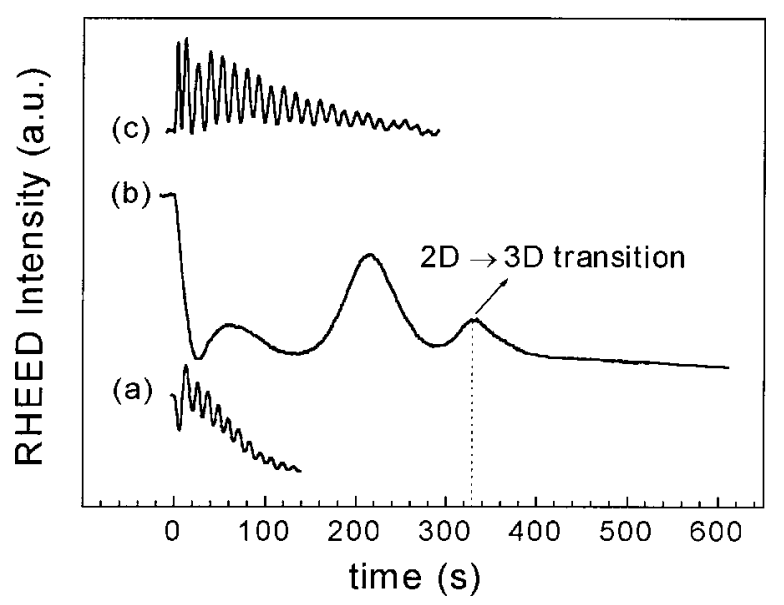

FIG. 1. RHEED specular $(0,0)$ beam intensity oscillations during heteroepitaxial growth of InN on $\mathrm{GaN}$ at (a) $T_{\text {sub }}=450{ }^{\circ} \mathrm{C}, \mathrm{In} / \mathrm{N}$ flux ratio $\sim 1.5$ and (b) $370{ }^{\circ} \mathrm{C}$ and $\mathrm{In} / \mathrm{N}$ ratio $\sim 0.6$, respectively; (c) represents RHEED oscillations during homoepitaxy of $\mathrm{GaN}$ at $T_{\mathrm{sub}}=450^{\circ} \mathrm{C}$, and $\mathrm{Ga} / \mathrm{N}$ ratio $\sim 2$.

is indicated by the initial layer-by-layer growth (RHEED intensity oscillates) followed by 3D islanding (RHEED intensity drops continuously). By changing the flux and temperature over a wide range of values, we establish that the SK mode is followed over all but one condition, i.e., under high In flux $(\mathrm{In} / \mathrm{N}>1)$ and high substrate temperature $\left(>420^{\circ} \mathrm{C}\right)$. Figure 2(a) shows a STM image of a surface following 7.5 BLs deposition under the latter condition. From the image, it is seen that a single BL high, triangularly shaped islands populate the entire surface. The triangular island shape is due to growth anisotropy of step edges of a wurtzite film. ${ }^{11}$ The orientations of islands on adjacent terraces are opposite while on the same terrace, they are the same. This morphology is a direct consequence of the hcp stacking of the lattice, confirming that the deposited $\mathrm{InN}$ layer is of pure wurtzite phase. ${ }^{11}$ Another observation in Fig. 2(a) is the presence of small pits. They are either inverted pyramids or hexagonal cones. Pit formation in InGaN layer has been reported previously and also studied theoretically. ${ }^{12,13}$ It was suggested that such pits could be initiated by threading dislocations or stacking mismatch boundaries in film. ${ }^{13}$ By counting the number of pits in image, we estimate the density of such defects is about $2 \times 10^{10} \mathrm{~cm}^{-2}$, which is consistent with transmission electron microscopy result for a GaN layer. ${ }^{14}$ Samples following the SK growth mode are also examined by STM. An example of such a surface is given in Fig. 2(b), which was prepared by growing $18 \mathrm{BLs} \mathrm{InN}$ at $350^{\circ} \mathrm{C}$ using

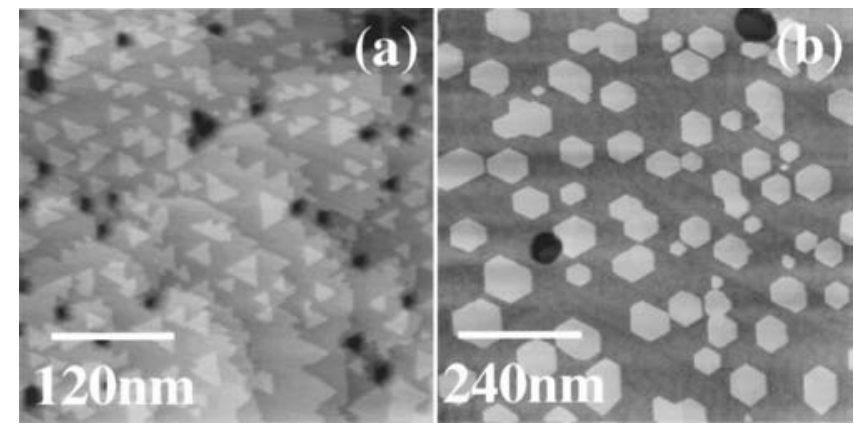

FIG. 2. STM image of InN surface following deposition at (a) $450{ }^{\circ} \mathrm{C}$ with In/ $\mathrm{N} \sim 1.5$ and (b) $350{ }^{\circ} \mathrm{C}$ with $\mathrm{In} / \mathrm{N} \sim 0.6$, showing, respectively, the $2 \mathrm{D}$ and $3 \mathrm{D}$ InN islands.



FIG. 3. Evolution of strain as a function of $\mathrm{InN}$ film thickness, calculated from the spacing $d$ (see inset) between $(01)$ and $(0 \overline{1})$ diffraction streaks of RHEED for (a) 2D and (b) SK growth, respectively. The inset show time evolution of the $d$ and the RHEED patterns of the starting GaN and finishing InN surfaces. The solid line in one of the RHEED images indicates the location where the $d$ is measured.

an excess-N flux ( $\mathrm{In} / \mathrm{N} \sim 0.6)$. From the image, one observes that 3D islands are well developed, which are hexagonal pillars with average height and lateral width being 5 and $84 \mathrm{~nm}$, respectively. It is, however, noted that the shape of 3D islands is affected by the flux of MBE. Mound-like islands are also obtainable if lower fluxes are used.

The observation of sustained 2D growth of $\mathrm{InN}$ film on $\mathrm{GaN}$ is surprising, as the system represents a large latticemismatch $(\sim 10 \%)$, in which case, 3D islanding would be preferred from both kinetic $^{15}$ and equilibrium considerations. ${ }^{16}$ In fact, 2D growth of $\mathrm{InN}$ has been previously observed by Nörenberg et al. ${ }^{6}$ however, there are also reports of the SK mode for the same system. ${ }^{7,8}$ This study reveals a surprising dependence of growth mode on the MBE condition, which explains the conflicting reports of the literature. In addition, the dependence of growth mode on MBE condition points to a kinetic origin of film growth mode, though the detail mechanism subjects to further investigation.

Having established film growth mode and morphology, we now turn our attention to the strain in film and its evolution as growth proceeds. To this end, the spacing $d$ between the neighboring integral diffraction streaks, namely $(0,1)$ and $(0, \overline{1})$ beams, of the RHEED is monitored. Obviously, $d$ represents the reciprocal of in-plane lattice parameter. Figure 3 shows, for both (a) 2D and (b) SK cases, the variation of strain $f$ as a function of the (nominal) thickness of deposited InN. The inset presents the evolution of $d$ as a function of growth time and the RHEED patterns obtained from the starting $\mathrm{GaN}$ and finishing InN surfaces. The strain $f$ is calculated according to $f \equiv\left(a_{\mathrm{InN}}-a_{\mathrm{epi}}\right) / a_{\mathrm{InN}}=[d(t)$ $-d(\infty)] / d(t)$, where $a_{\mathrm{InN}}=3.54 \AA$ is the lattice constant of a strain-free $\mathrm{InN}$ film, $a_{\mathrm{epi}}$ is that of the epitaxial $\mathrm{InN}, d(t)$ denotes the spacing between $(0,1)$ and $(0, \overline{1})$ diffraction beams at time $t$ measured experimentally while $d(\infty)$ denotes that after a long-time deposition when the strain is completely relieved (i.e., the reciprocal of InN lattice parameter). From Fig. 3, it is clear that strain relaxation commences upon the initiation (within the first BL) of film deposition 
and almost completes after 2-4 BLs growth. For the case of SK growth, 3D islanding occurs during the third BL deposition. This observation suggests that strain in the epitaxial InN is initially relieved by defects (e.g., dislocations) rather than by surface islanding. According to Matthews and Blakeslee's formula, ${ }^{17}$ it can be calculated that the critical thickness for dislocation formation in InN/GaN is indeed less than $1 \mathrm{BL}$. As is known, a competing mechanism for relieving the strain is by islanding. ${ }^{18}$ However, for $\mathrm{InN}$ on $\mathrm{GaN}$, islands form after a significant proportion of strain has been relieved and so they are likely dislocated and strain-free, consistent with the observation of Feuillet et al. ${ }^{7}$ Note that it is somewhat different from coherent 3D islands observed in other heteroepitaxial systems such as InGaAs on GaAs and SiGe on $\mathrm{Si}^{19,20}$ Rather, it conforms to the traditional, relaxed SK mode originally observed in metals. ${ }^{21}$ Another observation from Fig. 3 is that for the 2D growth, about $80 \%$ of the total strain is relieved within the first 2 BLs while the relaxation of the remaining strain is at a very slow rate. This contrasts to the case of SK growth, where strain relaxation proceeds continuously down to zero. However, surface islanding starts only after $80 \%$ of the total strain has been relieved by other mechanisms such as defect. It is thus likely that the latter stage strain-relieving mechanism is closely related to island formation.

To summarize, we have observed both $2 \mathrm{D}$ and $3 \mathrm{D}$ (SK) growth modes of InN films deposited on GaN by MBE. The growth mode is seen to be controlled by growth conditions: low temperature or high $\mathrm{N}$ flux (relative to $\mathrm{In}$ ) favors SK growth, whereas high temperature and high In flux leads to 2D growth. Strain in InN begins to relax upon the first layer deposition and almost completes after 2-4 BLs growth. For the case of SK growth, 3D islands form after most of the strain has been relieved and so the islands are likely dislocated and strain free.
This work is supported by grants from the Research Grants Council of the Hong Kong Special Administrative Region, China (Project Nos. 7118/02P and HKU7118/98P).

${ }^{1}$ S. Strite and H. Morkoç, J. Vac. Sci. Technol. B 10, 1237 (1992).

${ }^{2}$ S. Nakamura, M. Senoh, S. Nagahama, N. Iwasa, T. Yamada, T. Matsushita, Y. Sugimoto, and H. Kiyoku, Jpn. J. Appl. Phys., Part 2 36, L1059 (1997).

${ }^{3}$ M. Shimizu, K. Hiramatsu, and N. Sawaki, J. Cryst. Growth 145, 209 (1994).

${ }^{4}$ T. Böttcher, E. Einfeldt, V. Kirchner, S. Figge, H. Heinke, D. Hommel, H. Selke, and P. L. Ryder, Appl. Phys. Lett. 73, 3232 (1998).

${ }^{5}$ R. Singh, D. Doppalapudi, T. D. Moustakas, and L. T. Romano, Appl. Phys. Lett. 70, 1089 (1997)

${ }^{6}$ C. Nörenberg, M. G. Martin, R. A. Oliver, M. R. Castell, and G. A. D. Briggs, J. Phys. D 35, 615 (2002).

${ }^{7}$ G. Feuillet, B. Daudin, F. Widmann, J. L. Rouvière, and M. Arléry, J. Cryst. Growth 189/190, 142 (1998).

${ }^{8}$ W.-L. Chen, R. L. Gunshor, J. Han, K. Higashimine, and N. Otsuka, Mater. Res. Soc. Symp. Proc. 595, W3.30 (2000).

${ }^{9}$ S. M. Seutter, M. H. Xie, W. K. Zhu, L. X. Zheng, H. S. Wu, and S. Y. Tong, Surf. Sci. 445, L71 (2000).

${ }^{10}$ A. R. Smith, R. M. Feenstra, D. W. Greve, M.-S. Shin, M. Skowronski, J. Neugebauer, and J. E. Northrup, J. Vac. Sci. Technol. B 16, 2242 (1998).

${ }^{11}$ M. H. Xie, S. M. Seutter, W. K. Zhu, L. X. Zheng, H. S. Wu, and S. Y. Tong, Phys. Rev. Lett. 82, 2749 (1999).

${ }^{12}$ H. K. Cho, J. Y. Lee, G. M. Yang, and C. S. Kim, Appl. Phys. Lett. 79, 215 (2001).

${ }^{13}$ J. E. Northrup and J. Neugebauer, Phys. Rev. B 60, R8473 (1999).

${ }^{14}$ M. H. Xie, L. X. Zheng, S. H. Cheung, Y. F. Ng, H. S. Wu, and S. Y. Tong, Appl. Phys. Lett. 77, 1105 (2000).

${ }^{15}$ J. Tersoff and F. K. LeGoues, Phys. Rev. Lett. 72, 3570 (1994).

${ }^{16}$ C. Ratch and A. Zangwill, Surf. Sci. 293, 123 (1993).

${ }^{17}$ J. W. Matthews and A. E. Blakeslee, J. Cryst. Growth 27, 118 (1974); ibid. 29, 273 (1975); ibid. 32, 265 (1976).

${ }^{18}$ E. Bauer and J. H. van der Merwe, Phys. Rev. B 33, 3657 (1986).

${ }^{19}$ D. J. Eaglesham and M. Cerullo, Phys. Rev. Lett. 64, 1943 (1990).

${ }^{20}$ D. Vanderbilt and L. K. Wickham, in Evolution of Thin Film and Surface Microstructure, edited by C. V. Thompson, J. Y. Tsao, and D. J. Srolovitz (Material Research Society, Pittsburgh, 1991), p. 555.

${ }^{21}$ J. W. Matthews, D. C. Jackson, and A. Chambers, Thin Solid Films 26, 129 (1975). 
Applied Physics Letters is copyrighted by the American Institute of Physics (AIP). Redistribution of journal material is subject to the AIP online journal license and/or AIP copyright. For more information, see http:/ojps.aip.org/aplo/aplcr.jsp

Copyright of Applied Physics Letters is the property of American Institute of Physics and its content may not be copied or emailed to multiple sites or posted to a listserv without the copyright holder's express written permission. However, users may print, download, or email articles for individual use. 